\title{
MÉTODOS DE SUPERAÇÃO DA DORMÊNCIA DE SEMENTES DE PLANTAS DANINHAS DE PASTAGENS CULTIVADAS DA AMAZÔNIA1
}

\author{
ANTONIO P. da S. SOUZA FILHO ${ }^{2}$, SATURNINO DUTRA ${ }^{2}$ e MARIA A. M. M. SILVA ${ }^{3}$
}

\begin{abstract}
O objetivo do presente trabalho foi o de avaliar diferentes mé to dos de supe ração da dormência de sementes de plantas daninhas de áreas de pastagens cultivadas da região amazônica brasileira. Foram estudados os mé todos escarificação térmica em água a temperatu ra de $80^{\circ} \mathrm{C}$ por 4,8 e 12 minutos. escarificação química em ácido sulfúrico por 5, 10, 15 e 20 minutos e nitrato de potássio nas concentrações de 0,1: 0,2 e $0.3 \%$. As sementes, cuja dormência não foi superada por esses métodos, foram colocadas para germinar na presença de cinetina $(20,40,60$ e 80 ppm) e de giberelina (150. 300, 450 e 600 ppm). A germinação foi monitorada em períodos de 15 dias, com contagem diária e eliminação das sementes germinadas. A escarificação térmica em
\end{abstract}

RESUMO

água não se mostrou satisfatória, tendo havido, para a maioria das espécies, redução da germinação em relação ao tratamento testemunha. O ácido sulfúrico foi eficiente para superar a dormência das sementes de todas as espécies, havendo, no entanto, variações com relação ao tempo de imersão. O nitrato de potássio afetou positivamente a germinação das sementes de fedegoso, de rinchão e, mais expressivamente, de Hyptis mutabilis. As sementes de jurubebão responderam, positivamente, apenas aos diferentes níveis de giberelina. atingindo valor superior de germinação na concentração de 600 ppm.

Palavras chaves: Acido sulfúrico, água quente, nitrato de potássio. giberelina. cinetina.

\section{ABSTRACT \\ Dormancy overcoming methods of weed seeds from amazonian cultivated pasture}

The aim of this work was to evaluate different dormanc ${ }^{y}$ overcoming methods of weed seeds of cultivated pastures in the Amazon region. The following methods were studied: Thermal scarification in hot water $\left(80^{\circ} \mathrm{C}\right)$ for 4,8 and 12 minutes: chemical scarification in sulfuric acid for $5,10.15$ and 20 minutes and potassium nitrate in the concentrations of $0.1,0.2$ and $0.3 \%$. The seeds that did not overcome dormancy in these methods were put to germinate in the presence of kinetin $(20,40,60$ and $80 \mathrm{ppm})$ and gibberelic acid ( 150, 300. 450 and $600 \mathrm{ppm})$. Germination was monitorated during fifteen days, with daily counting and elimination of germinated seeds. Thermal sc ar if ic at ion in water was not a satisfactory procedure once it reduced germinability in relation to the control treatment. Scarification with sulfuric acid gave very good results. However, there were diferences according to the time of immersion.

The seeds germination of Cassis occidental is, Stachytarpheta cayennensis, and Hvptis mutabilis were affected $\mathrm{b}^{\mathrm{y}}$ potassium nitrate. Effects on seed germination of $H$. mutabilis were the most expressive. Solanum crinitum seeds responsed positively only to the different levels of gibberelic acid reaching a high value of germination at $600 \mathrm{ppm}$.

Key words: sulfuric acid, hot water, potassium nitrate, kinetin, gibberelic acid.

1 Recebido para publicação em 15/06/97 e na forma revisada em 04/02/9g.

2 Embrapa - Amazônia Oriental, Trav. Dr. Enéas Pinheiro, s/n, CEP: 66095-100, Belém/PA.

3 Professora da Faculdade de Ciências Agrárias do Pará, C.P. 917, CEP: 66077-530, Belém/PA.

Planta Daninha, v. 16, n. 1, 1998. 


\section{INTRODUÇÃO}

Um dos principais mecanismos de sobrevivência das plantas daninhas em ambientes constantemente perturbados. como os ecossistemas de pastagens cultivadas da região amazônica brasileira. é a alta produção de sementes (Carmona. 1995). Ao serem liberadas para o solo, entretanto muitas dessas sementes não germinam. embora viáveis e as condições ambientais neeessárias ao processo de germinação (luz. água. oxigênio) sejam. aparentemente. favoráveis. Nesse caso. as sementes são ditas dormentes.

A dormência das sementes pode ser atribuída a diferentes fatores. dentre os quais estão a impermeabilidade do tegumento à água e a gases. a imaturidade do embrião. a presença de inibidores ou ausência de promotores ou exigências espeeiais de luz ou temperatura (Vásques-Yanes \& OrozcoSagovia. 1993: Bewley \& Black. 1982). A impermeabilidade do tegumento. tipo de dormência bastante comum. tem sido constatado com frequência em sementes de Leguminosas, Malvaceae, Convolvulaceae e Solanaceae ( Popinigis. 1977: Cieero. 1986).

Com relação à superação da dormência das sementes que apresentam impermeabilidade do tegumento á água. diferentes procedimentos têm sido aplicados. com maior ou menor grau de sucesso. como a imersão em água quente e em ácido sulfúrico concentrado. por diferentes tempos (Toledo et al., 1993: Torres \& Santos. 1994). tendo sido obtidos resultados variáveis em função da espécie. Existe ainda um outro método de superação da dormência de sementes que envolve o emprego do nitrato de potássio. Perez \& Prado (1993) obtiveram excelentes resultados na germinação de sementes de Copaifera langsdorffii com tratamento envolvendo o nitrato de potássio a $2 \%$. A ação do nitrato. como agente de superação de dormência. parece decorrer de suas características oxidante e aceptor de elétrons, que possibilita o estímulo da via pentose fosfato, re su lt an do na ne ut ra li za çã o ou re du çã o da dormência das sementes (Roberts, 1972: Ellis et al., 1983).

O controle da germinação e da dormência, bem como de outros processos morfológicos da planta. tem sido, ultimamente, relacionado ao equilíbrio entre hormônios promotores e inibidores do erescimento (Amen. 1968). Conforme Khan (1975). a cinetina tem importante papel corno agente permissivo da ação da giberelina como promotor da germinação. Efeitos positivos da giberelina na germinação de sementes de diferentes espécies de plantas são encontrados nos trabalhos de Persson (1993) e de Laura et al. (1994).

$\mathrm{O}$ presente trabalho teve por objetivo avaliar diferentes métodos de superação da dormência de sementes de diferentes espécies de plantas daninhas que ocorrem em áreas de pastagens cultivadas da região amazôniea brasileira.

\section{MA TE RI AL E MÉ TO DOS}

Foram estudados diferentes métodos de superação da dormência de sementes das seguintes espécies de plantas daninhas: Fabaceae - Cassia tora L. (mata-pasto). Cassia occidenlalis L. (fedegoso) e Mimosa pudica Mill. (Malícia): Solanaceae - Solanum crinitum Lam. (jurubebão): Convolvulaceae - Ipomoea asarifolia Desr. (salsa): Poaceae - Paspahim virgatum Cham. \& Schlecht. (capim-navalha): Verbenaceae - Stachytarpheta cavennensis Vahl. (rinchão): e Lamiaceae - Hiptis mutabilis Brig. (sem nome comum).

As sementes foram coletadas em áreas de pastagens de Brachiaria humidicala e Brachiaria brizantha cv. Marandu, nos municípios de Castanhal e Paragominas. Estado do Pará. durante o ano de 1996. Foram submetidas. em seguida. ás operações de limpeza. de expurgo e de acondicionamento em câmaras de conservação de sementes sob umidade relativa e temperatura de $30 \%$ e IOC, respectivamente. Especificamente para o jurubebão. as sementes foram retiradas dos frutos, lavadas em água corrente, secas à sombra 
sob temperatura ambiente e acondicionadas a semelhança das demais.

Foram avaliados os seguintes métodos de superação da dormência:

a) escarificação térmica em água: consistiu na imersão das sementes em água à temperatura de 80 C. por 4.8 e 12 minutos, seguida de resfriamento e de secagem à sombra a temperatura ambiente:

b) escarificação química: as sementes foram mantidas imersas em ácido sulfúrico concentrado por 5. 10. 15 e 20 minutos. agitando-se ocasionalmente. com bastão de vidro: seguiu-se lavagem das sementes em água corrente. por idêntico tempo de imersão no ácido. e seca à sombra a temperatura ambiente:

c) nitrato de potássio: as sementes foram colocadas a germinar em papel de filtro umedecido com $6 \mathrm{ml}$ de nitrato de potássio nas concentrações de 0,1: 0,2 e $0.3 \%$, substituindo-se. diariamente. o papel de filtro por outro previamente umedecido com a mesma substância na concentração correspondente;

d) cinetina: empregado somente para as espécies cujas sementes não responderam aos métodos descritos nos itens "a", "b" e "c". Consistiu na colocação das sementes a germinar em papel de filtro umedecido com solução de cinetina, nas concentrações de 20, 40. 60 e 80 ppm. trocando-se diariamente. o papel de filtro por outro umedecido com cinetina na concentração correspondente:

e) giberelina: realizado apenas nas mesmas circuntâncias e de modo idêntico ao descrito no item "d". diferindo somente pela utilização de giberelina para unedecimento inicial, e quando das trocas diárias, do substrato, nas concentrações de 150, 300. 450 e 600 ppm.

Em todos os métodos empregados, a testemunha foi representada por sementes não submetidas a qualquer tratamento para superação da dormência.

Os efeitos dos tratamentos foram estimados mediante teste de germinação, conduzid o. por 15 dias, sob temperatura constante de $25^{\circ} \mathrm{C}$ e fotoperíodo de 12 horas de luz. Foram empregadas 50 sementes por repetição. dispostas sobre duas folhas de papel de filtro previamente autoclavadas a $120^{\circ} \mathrm{C}$ e umedecidas com água destilada ou solução correspondente. em caixas do tipo gerbox transparente, de $11 \mathrm{~cm}$ x $11 \mathrm{~cm}$. Foram realizadas. diariamente, contagens e eliminação das sementes germinadas. considerando-se como tal as que apresentavam extensão radicular igual ou superior a $2 \mathrm{~mm}$ (Juntila. 1976: Duran \& Tortosa. 1985).

O delineamento experimental utilizado em todos os ensaios foi o inteiramente casualizado com três repetições. sendo que. para cada espécie daninha. os tratamentos foram arranjados em esquema hierárquico com dois fatores: métodos de superação de dormência e níveis dentro de métodos. A análise da variância foi efetuada utilizando-se o sistema SAS, tendo por base o seguinte modelo matemático:

$$
Y i j k=m+A i+B j(i)+E i j k
$$

onde:

Yijk = variável de resposta medida nos

fatores $\mathrm{i}, \mathrm{j}$ e repetição $\mathrm{k}$ :

$\mathrm{m}=$ média geral;

$\mathrm{Ai}=$ efeito do fator $\mathrm{i}$ :

$\mathrm{Bj}(\mathrm{i})=$ efeito do fator $\mathrm{j}$ dentro do fator $\mathrm{i}$ :

Eijk = componente aleatório devido aos fatores $i, j$ e repetição $k$.

Para homogeneizar a variância entre tratamentos experimentais, utilizou-se a transformação angular nas variáveis de respostas medidas

\section{RESULTADOS E DISCUSSÃO}

\section{Efeitos da escarificação térmica}

As plantas daninhas estudadas apresentaram variações no grau de dormência das sementes. Assim, considerando o tratamento testemunha, sementes das espécies jurubebão, salsa e rinchão não germinaram, enquanto as espécies mata-pasto e $H$. mutabilis chegaram a apresentar 50,0 e $48.0 \%$ de germinação, respectivamente (Tabela 1).

As sementes das espécies manifestaram diferentes graus de sensibilidade ao calor. A 
tendência geral observada, entretanto, foi de redução ou perda do potencial de germinação com o aumento do tempo de imersão na água quente. As sementes de rinchão e de jurubebão não apresentaram germinação para qualquer dos tratamentos empregados. As de capim-navalha e de H. mutabilis mostraram-se mais sensíveis aos efeitos do calor, tendo o percentual de germinação dessas espécies, sido reduzido, respectivamente. de 29,0 e $48.0 \%$ para zero, quando as sementes permaneceram por . apenas. 4 minutos na água quente (Tabela 1).

TABELA 1. Efeito da escarificação térmica (água à 80" C) na germinação de sementes de plantas daninhas ocorrentes em áreas de pastagens cultivadas da Amazônia. Belém-PA, 1997.

\begin{tabular}{|c|c|c|c|c|c|}
\hline \multirow[t]{2}{*}{ Espicies } & & \multicolumn{4}{|c|}{ Tempo de imersão (minutos) } \\
\hline & & 0 & 4 & 8 & 12 \\
\hline \multirow[t]{2}{*}{ Malicia } & DO & 7,0 & 35.7 & 31.0 & 12.3 \\
\hline & DT & $0.280 \mathrm{c}$ & $0.650 \mathrm{a}$ & $0.601 \mathrm{a}$ & $0.373 b$ \\
\hline \multirow[t]{2}{*}{ Mata-Pasto } & DO & 50.0 & 52.0 & 43,0 & 20.0 \\
\hline & DT & $0.795 \mathrm{a}$ & $0.819 \mathrm{a}$ & $0.729 \mathrm{~b}$ & $0.475 \mathrm{c}$ \\
\hline \multirow[t]{2}{*}{ Fudegoso } & DO & 24,0 & 12.3 & 9.0 & 5.0 \\
\hline & DT & $0.523 \mathrm{a}$ & $0.373 \mathrm{~b}$ & $0.321 \mathrm{~b}$ & $0.245 \mathrm{c}$ \\
\hline \multirow{2}{*}{ Rinchão } & DO & 0.0 & 0.0 & 0.0 & 0.0 \\
\hline & DT & $0.100 \mathrm{a}$ & $0.100 \mathrm{a}$ & $0.100 \mathrm{a}$ & $0.100 \mathrm{a}$ \\
\hline \multirow[t]{2}{*}{ Salsa } & DO & 0.0 & 20.0 & 17.3 & 10.0 \\
\hline & DT & $0.100 \mathrm{c}$ & $0.476 a$ & $0.442 \mathrm{a}$ & $0.332 \mathrm{~b}$ \\
\hline \multirow[t]{2}{*}{ H. muabilis } & DO & 48.0 & 0.0 & 0.0 & 0.0 \\
\hline & DT & $0.775 \mathrm{a}$ & $0.100 \mathrm{~b}$ & $0.100 \mathrm{~b}$ & $0.100 \mathrm{~b}$ \\
\hline \multirow[t]{2}{*}{ Capim-Navalha } & DO & 29.0 & 0.0 & 0.0 & 0.0 \\
\hline & DT & $0.580 \mathrm{a}$ & $0.100 \mathrm{~b}$ & $0.100 \mathrm{~b}$ & $0.100 \mathrm{~b}$ \\
\hline \multirow[t]{2}{*}{ Jurubcbào } & DO & 0.0 & 0.0 & 0.0 & 0.0 \\
\hline & DT & $1.00 \mathrm{a}$ & $1.00 \mathrm{a}$ & $1.00 \mathrm{a}$ & $1.00 \mathrm{a}$ \\
\hline \multicolumn{6}{|l|}{$\begin{array}{l}\text { DMS }=0.053 \\
\mathrm{CV}=7.66 \%\end{array}$} \\
\hline
\end{tabular}

Das espécies de plantas daninhas estudadas. apenas malícia e salsa apresentaram acréscimos na germinação das sementes em resposta aos tratamentos, passando de $7.0 \%$ para $35,7 \%$ de germinação na malícia e de $0.0 \%$ para $20.0 \%$ de germinação na salsa. respectivamente nos tratamentos testemunha e imersão na água quente por 4 minutos (Tabela 1). Porém, esses acréscimos podem ser considerados baixos quando se entende a superação da dormência como um mecanismo de maximizar a germinação das sementes.

Apesar de ser um método vantajoso, quando se considera o baixo custo, os dados di sponíveis na literatura, à semelhança dos 6 resultados deste trabalho, mostram o emprego de água quente como método pouco satisfatório de superação da dormência de sementes com o tegumento duro (Rodrigues et al., 1990: Rodrigues \& Pitelli, 1994: Santarém \& Aquila. 199). embora alguns resultados positivos sejam encontrados, como é o caso do trabalho de Martins Neto (1994) que obteve germinação máxima de sementes de pau-de-balsa (Ochroma pyramidale), espécie com dormência associada à dureza do tegumento. através da imersão em água quente por 4 minutos.

A principal limitação ao emprego do calor, como método de superação da dormência de sementes, reside no fato de que tal procedimento

Planta Daninha, v. 16, n. 1, 1998. 
reduz a viabilidade das sementes, tanto através da morte como por danos provocados no embrião (Grus et al.. 1984). Mesmo quando em tempo de imersão em água quente inferior (1 e 2 minutos) ao do presente trabalho, tem sido relatada a morte de. praticamente, todas as sementes de espécies com dormência associada à impermeabilidade do tegumento (Maeda \& Lago, 1986).

\section{Efeitos da escarificação química}

Com exceção das sementes de jurubebão que não germinaram quando submetidas aos tratamentos com o ácido sulfúrico. as das demais plantas daninhas apresentaram acréscimos significativos $(\mathrm{P} \leq 0.05)$ no percentual de germinação em relação ao tratamento testemunha (Tabela 2).

As sementes das espécies estudadas atingi ram germinação máxima em diferentes tempos de imersão. com tendência a aumentos no percentual de germinação, com a elevação do tempo de imersão das sementes no ácido. A exceção foram as sementes de fedegoso. cujo percentual de germinação não diferiu $(\mathrm{P}>0.05)$ entre os diferentes tempos de imersão no ácido (Tabela 2).

TABELA 2. Efeito da escarificação química (ácido sulfúrico) na germinação de sementes de plantas daninhas ocorrentes em áreas de pastagens cultivadas da Amazônia. Belem-PA, 1997.

\begin{tabular}{|c|c|c|c|c|c|c|}
\hline \multirow[t]{2}{*}{ Especius } & & \multicolumn{5}{|c|}{ Tempo de imersão (minutos) } \\
\hline & & 0 & 5 & 10 & 15 & 20 \\
\hline \multirow[t]{2}{*}{ Malicia } & $\mathrm{DO}$ & 7.0 & 74.0 & 72.0 & 86.0 & 83,0 \\
\hline & DT & $0.280 \mathrm{c}$ & $1.048 \mathrm{~b}$ & $1.02 \mathrm{Ib}$ & $1.197 \mathrm{a}$ & $1.164 a$ \\
\hline \multirow[t]{2}{*}{ Mata-Pasto } & DO & 50.0 & 84.0 & 87,0 & 92,0 & 94.0 \\
\hline & DT & $0.795 \mathrm{c}$ & $1.17+b$ & $1.214 \mathrm{~b}$ & $1.300 \mathrm{a}$ & $1.355 \mathrm{a}$ \\
\hline \multirow[t]{2}{*}{ Fedegoso } & DO & 24.0 & 90.0 & 86.0 & 91.0 & 91.0 \\
\hline & DT & $0.522 \mathrm{~b}$ & $1,274 \mathrm{a}$ & $1.207 \mathrm{a}$ & $1.27 \mathrm{Ka}$ & $1.285 \mathrm{a}$ \\
\hline \multirow[t]{2}{*}{ Rinchào } & DO & 0.0 & 53.0 & 46.0 & 66.0 & 66.0 \\
\hline & DT & $0.100 \mathrm{c}$ & $0.829 \mathrm{~b}$ & $0.752 b$ & $0.955 \mathrm{a}$ & $0.955 a$ \\
\hline \multirow[t]{2}{*}{ Salsa } & DO & 0.0 & 28.0 & 47.0 & 44.0 & 650 \\
\hline & DT & $0.100 \mathrm{~d}$ & $0.568 \mathrm{c}$ & $0.762 \mathrm{~b}$ & $0,735 \mathrm{~b}$ & $0,952 \mathrm{a}$ \\
\hline \multirow[t]{2}{*}{ H. mulahilis } & DO & 48.0 & 89.0 & 52.0 & 390 & 23.0 \\
\hline & DT & $0.775 b$ & $1.255 \mathrm{a}$ & $0.815 b$ & $0.681 \mathrm{c}$ & $0.508 \mathrm{~d}$ \\
\hline \multirow[t]{2}{*}{ Capim-Navalha } & DO & 29.0 & 31.0 & 30,0 & 39.0 & 27.0 \\
\hline & DT & $0,580 \mathrm{~b}$ & $0.598 \mathrm{~b}$ & $0.590 \mathrm{~b}$ & $0.685 a$ & $0.56 \mathrm{lb}$ \\
\hline \multirow[t]{2}{*}{ Jurubcbào } & DO & 0.0 & 0.0 & 0.0 & 0,0 & 0.0 \\
\hline & DT & $0.100 \mathrm{a}$ & $0.100 \mathrm{a}$ & $0.100 \mathrm{a}$ & $0.100 \mathrm{a}$ & $0.100 \mathrm{a}$ \\
\hline \multicolumn{7}{|l|}{$\begin{array}{l}\text { DMS }=0.084 \\
\text { C. V }=5.09 \%\end{array}$} \\
\hline $\begin{array}{l}\text { Medias scguidas } \\
\text { DO = dados orig } \\
\text { DT = dados trans }\end{array}$ & is & . & $100+0.01$ & & 80 & \\
\hline
\end{tabular}

As sementes das espécies malícia. matapasto, rinchão e salsa apresentaram germinação máxima com os tempos de imersão no ácido de 15 e/ou 20 minutos. Considerando-se sementes de $H$. mutabilis. esse ponto foi atingido após 5 minutos de imersão, reduzindo-se, a partir de então, com o aumento do tempo de imersão das sementes, sendo os percentuais de germinação obtidos aos 15 $(39,0 \%)$ e aos $20(23,0 \%)$ minutos de imersão. inferiores $(\mathrm{P} \leq 0,05)$ aos observados para $\mathrm{o}$ tratamento testemunha $(4,0 \%)$ (Tabela 2$)$.

$\mathrm{O}$ ácido sulfúrico tem sido usado para promover a germinação tanto de sementes com te gu mento impermeável à água qu an to de 
sementes cujos tecidos de cobertura, embora permeáveis à água. evitam ou retardam a germinação, como ocorre frequentemente nas gramíneas (Ellis et al., 1985). Neste trabalho foram observados efeitos positivos do ácido sulfúrico na germinação das sementes de capimnavalha. destacando-se o tempo de 15 minutos de imersão no ácido ao promover acréscimo de $34,4 \%$ no percentual de germinação. em relação ao tratamento testemunha.

Os acréscimos verificados na germinação das sementes das plantas daninhas estudadas neste trabalho. quando empregou-se o ácido sulfúrico para superação da dormência, são semelhantes aos obtidos por diferentes autores como Maeda \& Lago (1986): Martins et al.
(1992): Eira et al. (1993): Danthu et al. (1995).

\section{Efeitos do nitrato de potássio}

À semelhança dos resultados observados quando do emprego da água quente (Tabela 1) e do ácido sulfúrico (Tabela 2). as sementes de jurubebão novamente não apresentaram germinação na presença e na ausência do nitrato de potássio (Tabela 3). Conquanto diferenças significativas $(\mathrm{P} \leq 0,05)$ tenham sido observadas. para algumas espécies entre os tratamentos envolvendo o uso do nitrato de potássio e a testemunha, os acréscimos verificados no percentual de germinação foram relativamente baixos. quando consideram-se aqueles obtidos com ácido sulfúrico (Tabela 2).

TABELA 3. Efeito do nitrato de potássio na germinação de sementes de plantas daninhas ocorrentes em áreas de pastagens cultivadas da Amazônia. Belém-PA. 1997.

\begin{tabular}{|c|c|c|c|c|c|}
\hline \multirow[t]{2}{*}{ Especies } & & \multicolumn{4}{|c|}{ Concentração (\%) } \\
\hline & & 0.0 & 0.1 & 0.2 & 0.3 \\
\hline \multirow[t]{2}{*}{ Malicia } & $\mathrm{DO}$ & 7.0 & 10,0 & 9.0 & 7.0 \\
\hline & DT & $0,280 \mathrm{~b}$ & $0.330 \mathrm{a}$ & $0.322 b$ & $0.280 \mathrm{~b}$ \\
\hline \multirow[t]{2}{*}{ Mata-Pasto } & DO & 50.0 & 39.0 & 50.0 & 47.0 \\
\hline & DT & $0.795 \mathrm{a}$ & $0.685 \mathrm{~b}$ & $0.795 a$ & $0.763 \mathrm{a}$ \\
\hline \multirow[t]{2}{*}{ Fedegoso } & $\mathrm{DO}$ & 24.0 & 18.0 & 33.0 & 25.0 \\
\hline & DT & $0.522 \mathrm{~b}$ & $0.45 \mathrm{lc}$ & $0.622 \mathrm{a}$ & $0.535 \mathrm{~b}$ \\
\hline \multirow[t]{2}{*}{ Rinchào } & DO & 0.0 & 21.0 & 19.0 & 14.0 \\
\hline & DT & $0.100 \mathrm{c}$ & $0.484 a$ & $0.463 \mathrm{a}$ & $0.397 \mathrm{~b}$ \\
\hline \multirow[t]{2}{*}{ Salsa } & $\mathrm{DO}$ & 0.0 & 4.0 & 4.0 & 3,0 \\
\hline & DT & $0.100 \mathrm{~b}$ & $0.218 \mathrm{a}$ & $0.226 \mathrm{a}$ & $0.209 \mathrm{a}$ \\
\hline \multirow[t]{2}{*}{ H. mulotilis } & DO & $4 \times 0$ & 67.0 & 67.0 & 87,0 \\
\hline & $\mathrm{DT}$ & $0.775 c$ & $0.467 \mathrm{~b}$ & $0.977 \mathrm{~b}$ & $1.224 \mathrm{a}$ \\
\hline \multirow[t]{2}{*}{ Capim-Navalha } & DO & 29.0 & 24.0 & 22.0 & 26.0 \\
\hline & $\mathrm{DT}$ & $0.580 \mathrm{a}$ & $0.531 \mathrm{a}$ & $0.504 \mathrm{a}$ & $0.543 \mathrm{a}$ \\
\hline \multirow[t]{2}{*}{ Jurubcbào } & $\mathrm{DO}$ & 0.0 & 0,0 & 0,0 & 0.0 \\
\hline & DT & $0.100 \mathrm{a}$ & $0.100 \mathrm{a}$ & $0.100 \mathrm{a}$ & $0.100 \mathrm{a}$ \\
\hline
\end{tabular}

DMS $=0.05$

C. V $=4.84 \%$

Médias seguidas de letras iguais na linha. nào diferem entre si pelo teste de Tukẹ $(P>0,05)$

DO = dados originais ( $\%)$

DT $=$ dados transformados para arc sen $\sqrt{ } / 100+0.01$

O nitrato é citado como o único ion inorgânico comum na solução do solo que afeta a germinação de sementes de urna vasta gama de espécies (Espeby, 1989). No presente trabalho, estie efeito também foi observado, em especial para as invasoras fedegoso, rinchão e $H$. mutabilis 
(Tabela 3). Maiores porcentagens de germinação de sementes de fedegoso e de rinchão foram obtidas quando utilizou-se concentrações de nitrato de potássio de $0.2 \%$ e $0.1 \%$, respectivamente.

Das espécies estudadas, o H. mutabilis foi a que apresentou os maiores acréscimos no percentual de germinação das sementes. passando de $48 \%$ (tratamento testemunha) para $87 \%$. quando na presença do nitrato de potássio na concentração de $0.3 \%$. correspondendo a um aumento da ordem de $81.3 \%$. Este valor está bem próximo daquele obtido após imersão das sementes da mesma espéeie em ácido sulfúrico por 5 minutos que foi de $89,0 \%$ (Tabela 2). indicando a possibilidade de utilização de ambos os métodos para maximização da germinação das sementes.

$\mathrm{O}$ nitrato de potássio tem sido indicado como estimulador da germinação de sementes de gramíneas na concentração de $0.2 \%$. para condições semelhantes ao deste ensaio (West \& Marousky. 1989: Brasil. 1992). De acordo com os dados apresentados na Tabela 3. esta recomendação não se mostrou efetiva para o capim-navalha. que não respondeu $(\mathrm{P}>0.05)$ às diferentes concentrações do nitrato de potássio.
Outros resultados encontrados por diferentes autores também não mostraram efeitos positivo, do nitrato de potássio na germinação de sementes de gramíneas. como é o caso dos trabalhos de Huang \& Hsiano (1987) e de Gazziero el al (1991).

\section{Efeitos da cinetina e da giberelina}

Das espécies de plantas daninhas estudadas. o jurubebão foi a única cuja germinação das sementes não foi afetada por qualquer dos métodos de superação da dormência testados. Esse resultado indica que. provavelmente. a dormência das sementes dessa invasora esteia associada a outros fatores que não à impermeabilidade do tegumento.

Em função desse aspecto. as sementes de jurubebão foram colocadas para germinar em papel de filtro umedecido com cinetina. nas concentrações de 20. 40, 60 e 80 ppm. Após um período de 15 dias de observação. não foi verifiada germinação das serventes.

Nos testes envolvendo o emprego da giberelina. nas concentrações de 150, 300, 450 e $600 \mathrm{ppm}$, indicaram efeitos $(\mathrm{P} / * \backslash 0,05)$ positivos sobre a germinação das sementes de jurubebão (Tabela 4).

TABELA 4. Efeito da giberelina na germinação de sementes de plantas daninhas ocorrentes em áreas de pastagens cultivadas da Amazônia. Belém-PA. 1997.

\begin{tabular}{|c|c|c|c|c|c|c|}
\hline \multirow[t]{2}{*}{ Espicic } & & \multicolumn{5}{|c|}{ Concentraçio ( ppm) } \\
\hline & & 0.0 & 150 & 300 & 450 & 600 \\
\hline \multirow[t]{2}{*}{ Jurubcbão } & DO & 0.0 & 20,0 & 34.0 & 47.0 & 52.0 \\
\hline & DT & $0.100 \mathrm{c}$ & $0.476 \mathrm{~d}$ & $0.633 \mathrm{c}$ & $0.769 \mathrm{~b}$ & $0.822 \mathrm{a}$ \\
\hline
\end{tabular}

Medias seguidas de letras iguais na linha. não diferem entre si pelo teste de Tukey $(P>0,05)$.

DO $=$ Dados originais $(\%)$

DT $=$ Dados transformados para arc sen $\sqrt{ } / 100+0,01$

Os dados mostram que o percentual de germinação foi crescente com o aumento da concentração de giberelina tendo atingido o valor máximo de germinação $(52,0 \%)$ na concentração de $600 \mathrm{ppm}$. Entretanto. aparentemente as concentrações de giberelina te stad as foram insuficientes para maximizar a germinação das sementes de jurubebão, havendo, portanto, a necessidade de estudos envolvendo concentrações mais elevadas de giberelina, com vista a obtenção de valores mais satisfatórios de germinação. 
O uso exclusivo do papel da giberelina como hormônio promotor primário da germinação, conforme observado no presente trabalho. tem sido demonstrado por vários autores como Karssen et al. (1989) e Cunha (1989).

\section{LITERATURA CITADA}

AMEN. R D A model of sed dormancy. Bot Ver., 1.34.n.1. p. 1-30. 1968

BEWLEY. J.D. BLACK. M. Physiology and biochemistry of seeds in relation to germination. Vol.2. Berlin: SpringerVerlag. $1982.378 \mathrm{p}$

BRASIL Ministerio da Agricultura Regras para Análise de Sementes. Brasilia: Departamento Nacional de Produção Vegetal. Divisão de Sementes è Mudas 1992. $365 \mathrm{p}$

CARMONA. R. Banco de sementes e estabelecimento de plantas daninhas $\mathrm{cm}$ agroccossistemas. Planta Daninha. Y.13. n.1. p.3-9.1995.

CICERO, SM Dormencia de sementes. In SEMANA DE ATUALIZAÇ̃O EM PRODUÇÃO DE SEMENTES, 1.. 1986. Piracicaba Anais... Campinas Fundação Cargill. 1986 p.41-73

CUNHA. R. CASALI, V.W.D. Efeito de substâncias reguladoras de crescimento sobre a germinaçào de sementes de alface. R. Bras. Fisiol. Veg., v.I. n.2. p.121-132. 1489.

DANTHU, P.. ROUSSEL. J. GAYE. A. . MAZZOUDI. E.L Baobab (Adansona digitata L.) seed pretreatments for germination improvement. Seed Sci. Technol., v.23, n.2. p. 469-475. 1995.

DURAN, JM. TORTOSA, M.E. The effect of mechanical and chemical scarification on germination of charlock (Sinapis arvensis L.) seeds. Seed Sci. Technol., v.13, n.1. p.155-163.1985

EIRA. M T. S., FREITAS. R. W A. MELLO. C. M. C. Superação da dormêneia de sementes de Enterolobium contortisiliqum (Vell) Morong - Leguminosac R. Brasi. Sem.. ज15.n.2, p.177-181.1993

ELLIS, R.H. HONG. T.D.. ROBERTS, EH. Procedures for the safe removed of dormancy from rice seed. Seed Sci. Technol.. v.1I.n. I. p.77-112. 1983

ELLIS. R H. HONG, T.D., ROBERTS. E. H. Handbook of seed technology for genebanks, compendium of specific germination information and test recommendation. Rome: IBPGR. 1985. v. 2 . p. $211-667$

ESPEBY. L. Germination of weed seeds and competition in stands of weeds and barley influence of mineral nutrients. Crop Prod. Sci. v 6, p.1-172, 1989

GAZZIERO, D. L. P., KZRYZANOWSKI, F. C.. ULBRICH, A. V., VOLL, E., PITELLI, R. A. Estudo da superação de dormência de sementes de capim-massambara (Sorghum halepense (L.) Pers.), atraves de nitrato de potássio e ácido sulfúrico. R. Bras. Sem. v.13.n. I. p.2I-24. 199I

GRUS, V. M. DEMATE, M. E. S P GRAZIANO. T J Germinação de sementes de pau-ferro e cassia-javanesa submetida a tratamento para quebra de dormência. R. Bras. Sem.. v.6, n.2, p.2935. 1984

HUANG, W. Z.. HSIANO. A. I Factors affeting seed dormancy and germination of johnsongrass (Sorghum halepensix (L.) Pers.). Weed Res., v.27, p.1-12, 1987

IUNTILA. O. Seed and embryo germination in S. vulgaris and $S$ reflexa as effected by temperature during seed development Physiol. Plant., v.29. p. 264-268, 1976. 
KARSSEN, C M. ZAGORSKI S. KEPCZYNSKI. J., GROOT. S. P. C. Ku role for endogenous giberelina in the control seed germination. Ann. Bot.. v 63. p $71-80.1989$

KHAN. A A Primary preventive and permissive roles of hormones in plant systems. Bot. Rev.. v. 41. p. 391-420. 1975

LAURA. V. A. ALVARENGA. A. A.. ARRIGON. MF. Effects of growth regulators, temperature, light. storage and others factors on the Monninga culubura L sced germination. Seed Sci. Technol.. 1.22, n.3.p. $573-579.1994$

MAEDA, J A , LAGO. A A Germinação de sementes de mucuna-preta apos tratamento para superação da impermeabilidade do tegumento. R. Bras. Sem. v.8, n.1. p.7984.1986

MARIINS. C. C.. CARVALHO. N. M. OLIVEIRA. A. P. Quebra de dormência de sementes de sabia (Mimosa caesalpunactolia Benth). R. Bras. Sem. v.14.n.1.p.5-8. 1992

MARTINS NETO. D A Germinaçào de sementes de pau-de-balsa (Ochroma plramidale (Cas.) Urb) - Bombacaceac R. Bras. Sem.. v.16, n.2. p.159-162, 1994

PEREZ S C. J G. PRADO. C. H. B. A Efeitos de diferentes tratamentos pré-germinativos e da concentração de aluminio no processo germinativo de sementes de (opaifera langsdertfii Desf. R. Bras. Sem., v 15, n. I. p.115-118. 1993

PERSSON. B. Enhancement of seed germination in ornamental plant by growth regulators insufed via acetone. Seed Sci. Technol. 1.21.n.2. p.281-290.1993
POPINIGIS, F Fisiologia de sementes. Brasilia AGIPLAN, 1977. 289p.

ROBERTS. E.H. Oxidative processes and the control of seed germination. In: HEYDECKER. W. ad. Seed Ecology Universits Park: Pennsilvania Statc University Press. 1972 p.189-218

RODRIGUES, E. H. A ., AGUIAR. I. B.. SADER. R. Quebra de dormência de sementes de très espécies do gènero ('assia. R. Bras. Sem. 1.12, n.2. p.17-27. 1990

RODRIGUES, B N PITELLI, R A Quebra de dormência $\mathrm{em}$ sementes de Commelina henghalensis. Planta Daninha, v.12. n.2. p.106-110.1994

SANTAREM. E. R. AQUILA. M.E.A Influência de métodos de superação de dormência e do armazenamento, na germinaçào de sementes de Senna macranthera (colladon) Inum \& Barneby (Leguminosac). R. Bras. Sem. v.17.n.2. p.205-209. 1995

TOLEDO, R. E. B., KUVA, M A ALVES. P L. C. A Fatores que afetam a germinaçào e a emergência de Kanthsum strumarium L dormència. qualidade da luz e profundidade de semeadura Planta Daninha. I.II. n. 1/2. p. 15-20, 1993

TORRES. S. B. SANTOS. D. S. B. Superação de dormência em sementes de Acacta senegal (L.) Willd e Parkinsonia aculenta (L.) R. Bras. Sem., v. 16. n. I. p. 54-57. 1994

VASQUES-YANES, C. OROZCO-SAGOVIA. A. Patherns of seed longevity and germination in the tropical rain forest Ann. Rev. Ecol. Syst.. v.24. p.69-87. 1993

WEST. 3.H. MAROUSKY. F. Mechanism of dormancy in Pensacola Bahiagrass. Crop Sci. v.29.n.3.p.781-791. 1989 